\title{
Correlación entre los puntajes del Wisconsin Card Sorting Test y la actividad electroncefalográfica en mujeres en la posmenopausia
}

\author{
Correlation between the scores of the Wisconsin Card Sorting Test and electroencephalographic \\ activity in postmenopausal women
}

\author{
Silvia Solís-Ortiz ${ }^{1 *}$, Mayra Gutiérrez ${ }^{2}$ \\ 1 Departamento de Ciencias Médicas de la División de Ciencias de la Salud, Campus León, Universidad de Guanajuato. Guanajuato, \\ México. \\ 2 Universidad de Guanajuato. Guanajuato, México.
}

\section{Resumen}

El objetivo fue correlacionar los puntajes obtenidos en la ejecución de la prueba Wisconsin Card Sorting Test con las medidas de la actividad electroencefalográfica (EEG) en 48 mujeres entre 48 y 65 años de edad en la posmenopausia. La actividad EEG fue registrada en reposo en F3, F4, F7, F8, FC3, FC4, C3, C4, CP3, CP4, P3, P4, O1, O2, FT7, FT8, Fz, Cz, CPz, Pz y Oz. Se obtuvo la potencia de delta, theta, alfa1, alfa2, beta1 y beta2 y se correlacionaron con el número de categorías completadas, cartas presentadas, errores cometidos, errores perseverativos, número de ensayos y errores a las categorías de la prueba. La banda de beta 2 se correlacionó positivamente con el número de categorías completadas $(p=0.04)$ y negativamente con el número de errores perseverativos $(p=0.03$ ) y con el número de errores cometidos $(p=0.03)$. Estos resultados indican que un estado de activación cortical de frecuencias rápidas es necesario para ejecutar exitosamente pruebas que involucren funciones ejecutivas en mujeres con bajos niveles de estrógenos debidos a la posmenopausia.

Palabras clave: Wisconsin Card Sorting Test, funciones ejecutivas, cognición, menopausia, electroencefalografía

\begin{abstract}
The aim was to correlate the scores of the performance of Wisconsin Card Sorting Test with measures of electroencephalographic activity (EEG) in 48 postmenopausal women between 48 and 65 years. EEG activity was recorded at rest in F3, F4, F7, F8, FC3, FC4, C3, C4, CP3, CP4, P3, P4, O1, O2, FT7, FT8, Fz, Cz, CPz, Pz and Oz. The power of delta, theta, alpha1, alpha2, beta1 and beta2 was obtained and correlated with the number of categories completed, cards presented, errors, perseverative errors, number of trials and errors to the categories. Beta2 band was positively correlated with the number of categories completed $(p=0.04)$ and negatively with the number of perseverative errors $(p=0.03)$ and the number of errors $(p=0.03)$. These results indicate that a state of cortical activation fast is necessary to successful performance in tests that involve executive function in women with low estrogen levels due to postmenopause.
\end{abstract}

Keywords: Wisconsin Card Sorting Test, executive functions, cognition, menopause, electroencephalographic

\footnotetext{
* Correspondencia: silviasolis17@prodigy.net.mx. Departamento de Ciencias Médicas, Universidad de Guanajuato. 20 de Enero 929 Colonia Obregón, León, Guanajuato, CP 37350, México. Teléfono: +52 (477) 7143812 extensión 16. Fax: +52 (477) 7167623

Recibido: 02-11-11. Revisión desde: 04-11-11. Aceptado: 27-12-11

DOI: $10.5839 / \mathrm{rcnp} .2011 .0602 .03$
}

Copyright $\odot 2011$ Universidad de La Frontera ISSN 0718-4913 (versión en línea) 


\section{Introducción}

El Wisconsin Card Sorting Test (WCST) (Heaton, 1981) es una prueba neuropsicológica estándar que mide funciones ejecutivas. La ejecución del WCST activa varias regiones cerebrales, que incluyen la corteza prefrontal izquierda, el lóbulo parietal inferior y la porción posterior de la corteza temporal inferior (Berman, Ostrem, Randolph, Gold, Goldberg, Cappola, et al., 1995). Esta prueba involucra la evaluación de habilidades relacionadas con razonamiento abstracto y el uso de estrategias cognitivas para la resolución de problemas, planeación, memoria de trabajo, organización y conducta dirigida a una meta (Heaton, Chelune, Taley, Kay \& Curtiss, 1993). Todas estas funciones son conocidas como funciones ejecutivas atribuidas a la corteza prefrontal (Nauta, 1971; Stuss, Benson, Clermont, Della Malva, Kaplan \& Weir, 1986; GoldmanRakic, 1987; Fuster, Bauer \& Jervey, 1985; Petrides, 1991; Damasio, 1993).

Las funciones ejecutivas tienden a variar con la edad y con el estado hormonal de las mujeres. Se ha observado una mejor ejecución del WCST en la fase lútea del ciclo menstrual en mujeres jóvenes, indicado por el menor número de errores cometidos y mayor número de categorías alcanzadas en la prueba (Solís-Ortiz, Guevara \& Corsi-Cabrera, 2004). Deficiencias cognitivas como memoria pobre, incapacidad para concentrarse, deficiencias en el razonamiento abstracto, atención y flexibilidad cognitiva se han reportado en mujeres posmenopáusicas de mediana edad (Sullivan \& Fugate, 2001; Tivis, 1999; Halbreich, Lumley, Palter, Manning, Gengo \& Joe, 1995; Sepúlveda, Pérez \& Solís, 2009). La relación de las funciones ejecutivas medidas con la prueba WCST con la actividad electroencefalográfica (EEG) en la posmenopausia se conoce poco. El electroencefalograma mide la actividad eléctrica en el cerebro con alta resolución temporal en el tiempo, registrada en periodos cortos de tiempo, y proporciona una medida directa del estado funcional del cerebro y de sus niveles diferentes de activación (Neidermeyer, 1993; Buzsaki, 2006). Los patrones de actividad EEG reflejan la activación cortical, funciones cognitivas y estados de conciencia. Esta técnica funcional no invasiva típicamente requiere de pocos minutos a una hora de la participación de los sujetos para adquirir grupo de datos sustanciales de un solo sujeto (Reilly, 1993). Aunque la lectura de la actividad EEG es compleja y varía en muchas dimensiones, incluyendo la distribución de las frecuencias, amplitud en varias regiones del cerebro y morfología de las ondas cerebrales, las características individuales tienden a ser estables (Salinsky, Oken \& Morehead, 1991; Corsi-Cabrera, Solís-Ortiz \& Guevara, 1997; Corsi-Cabrera, Galindo-Vilchis, del-Río-Portilla, Arce \& Ramos-Loyo, 2007). Existe evidencia que muestra que la actividad EEG en reposo es afectada por cambios hormonales en los niveles de hormonas sexuales durante el ciclo menstrual en mujeres jóvenes (Becker, Creutzfeldt, Schwibbe \& Wuttke, 1982; Solís-Ortiz, Ramos, Arce, Guevara \& Corsi-Cabrera, 1994) y que permite una evaluación de las fluctuaciones de la actividad y su correlación con la cognición cuando las hormonas son bajas, como en la posmenopausia. La relación de las funciones ejecutivas con la actividad electroencefalográfica (EEG) en la posmenopausia se conoce poco. El objetivo del presente estudio fue correlacionar los puntajes obtenidos en la ejecución de la prueba WCST con las medidas de la actividad EEG obtenidas en reposo en un grupo de mujeres en la posmenopausia.

\section{Método}

Sujetos

Un total de 48 mujeres voluntarias, en la posmenopausia, entre 48 y 65 años de edad, participaron en un estudio de sección transversal. Este tamaño de muestra fue calculado para un poder estadístico esperado de 0.86 , con el fin de detectar un $10 \%$ de diferencia en la ejecución cognitiva de la prueba a dos colas con un nivel de significancia de $\alpha=0.05$. A todas las mujeres se les realizó una entrevista clínica para evaluar su estado de salud. Las mujeres que participaron en el estudio tuvieron por lo menos 12 meses de amenorrea, sin historia de enfermedad cardiovascular, metabólica, endocrinológica o cáncer. Ninguna de las participantes estaba tomando terapia de reemplazo hormonal o medicamentos. La demencia incipiente fue evaluada con la prueba Mini-Mental (Folstein, Folstein \& McHugh, 1975). Los puntajes de esta prueba van de los rangos de 0 a 30 y los sujetos con demencia generalmente muestran puntajes debajo de 24. En el presente estudio, los puntajes de la prueba estuvieron de 27 a 30 . Las participantes fueron evaluadas en una sola sesión por una psicóloga entrenada entre las 9:00 y 11:00 horas. Se instruyó a las participantes de abstenerse de tomar café, fumar, beber alcohol, y de dormir ocho horas el día previo al estudio. Este estudio fue aprobado por el Comité de Ética del Departamento de Ciencias Médicas de la Universidad de Guanajuato para la investigación en sujetos humanos y está de acuerdo con la Declaración de Helsinki. Todas las participantes dieron su consentimiento informado por escrito antes de participar en el estudio.

\section{Wisconsin Card Sorting Test (WCST)}

Una prueba neuropsicológica estandarizada, el WCST (Heaton, 1981), en versión computarizada, se utilizó para evaluar las funciones ejecutivas prefrontales. EI WCST activa varias regiones cerebrales, incluyendo la corteza dorsolateral prefrontal, el lóbulo parietal inferior y la porción posterior de la corteza temporal inferior (Berman, Ostrem, Randolph, Gold, Goldberg, Coppola, et al., 1995). El WCST es una prueba de razonamiento abstracto y resolución de problemas, que requiere del uso de la memoria de trabajo para formar un proceso cognitivo y aplicar una estrategia. Esta prueba también necesita mantener y adaptar el proceso cognitivo cuando es necesario. Los estímulos fueron presentados en una pantalla en frente de los sujetos. EI WCST requiere que los sujetos descubran la regla para aparear cartas. A los sujetos se les presenta un grupo de cartas que contienen figuras geométricas (triángulos, estrellas, cruces y círculos) que varían en color (rojo, verde, azul o amarillo) y números $(1,2,3$, y 4$)$, y deben descubrir la regla para aparear cartas. Los sujetos deben asignar, donde consideren que debe ir, la carta correspondiente - que se presenta en el extremo inferior derecho de la pantalla - a una de las cuatro cartas que se encuentran en el extremo superior de la pantalla. Una vez seleccionada la carta, si el criterio de clasificación para la carta es el color, el sujeto asignará la carta a la correspondiente al color y aparecerá en la pantalla la palabra "correcto", de lo contrario aparecerá la palabra "incorrecto". El criterio de clasifi- 
Solís-Ortíz, Gutiérrez. Rev. chil. neuropsicol. 6(2): 76-79, 2011

Tabla 1. Correlación entre los puntajes de la prueba Wisconsin Card Sorting Test y las bandas de la actividad electroencefalográfica.

\begin{tabular}{|c|c|c|c|c|c|c|}
\hline \multirow[t]{2}{*}{ Variables del Wisconsin Card Sorting Test } & \multicolumn{6}{|c|}{ Bandas de la actividad electroencefalografía } \\
\hline & Delta & Theta & Alfa1 & Alfa2 & Beta1 & Beta2 \\
\hline Número de cartas & -0.04 & 0.08 & -0.03 & 0.04 & -0.10 & -0.33 \\
\hline Categorías completadas & 0.13 & -0.07 & 0.07 & 0.01 & 0.01 & $0.36 *$ \\
\hline Errores perseverativos & 0.01 & 0.17 & 0.09 & 0.10 & -0.02 & $-0.37 *$ \\
\hline Errores & -0.09 & 0.18 & 0.07 & 0.12 & 0.04 & $-0.37 *$ \\
\hline Errores a las categorías & -0.10 & -0.20 & -0.14 & -0.15 & -0.04 & -0.16 \\
\hline Respuestas correctas & -0.19 & -0.14 & -0.24 & -0.22 & -0.19 & -0.24 \\
\hline
\end{tabular}

$* p<0.05, R$ de Sperman

cación cambia aleatoriamente y el sujeto debe descubrir esto conforme avanza la prueba. La primera categoría es el criterio de selección al color, que continúa hasta que el sujeto completa 10 selecciones consecutivas del criterio. Posteriormente, el criterio cambia a selección de figura, sin previo aviso, y el sujeto debe descubrir el cambio y hacer las selecciones de acuerdo con la figura y así sucesivamente hasta que complete seis categorías correctas (dos de cada criterio) o hasta que se hayan presentado 128 cartas. Los siguientes resultados provistos por la versión computarizada fueron analizados: (1) número de categorías completadas; (2) el número de respuestas correctas (ensayos); (3) errores perseverativos; (4) el número de errores; (5) errores cometidos a las categorías; (6) número de cartas presentadas.

\section{Registro de la actividad electroencefalográfica}

La actividad electroencefalográfica (EEG) fue registrada entre las 9:00 y 11:00 horas en reposo. Veintidós electrodos fueron colocados de acuerdo al Sistema 10-20 Internacional en F3, F4, F7, F8, FC3, FC4, C3, C4, CP3, CP4, P3, P4, O1, O2, FT7, FT8, Fz, $\mathrm{Cz}, \mathrm{CPz}, \mathrm{Pz}, \mathrm{Oz}$ usando una gorra modelo Quik Cap. La actividad EEG fue registrada en un amplificador NuAmps digital de 40 canales modelo Neuroscan con los filtros de frecuencia de 0.5 a $35 \mathrm{~Hz}$. La actividad EEG fue grabada en una computadora personal con una frecuencia de muestreo de $512 \mathrm{~Hz}$ y fue analizada fuera de línea con el programa SCAN 4.3. La actividad EEG fue cuidadosamente inspeccionada para eliminar del análisis los artefactos de movimientos de ojos o signos de sueño. Los participantes fueron instruidos a que se relajaran y sentaran en una silla confortable para el registro en reposo. La sesión de registro consistió de tres minutos con los ojos abiertos y tres minutos con los ojos cerrados. Un mínimo de 20 épocas libres de artefactos, de cada dos segundos de duración, fueron analizadas con la Transformada Rápida de Fourier. La potencia absoluta $(\mathrm{Hz})$ fue obtenida para la siguientes bandas: delta, 0.5 a $4.0 \mathrm{~Hz}$; theta, $4.0-8.0 \mathrm{~Hz}$; alfa1, $8.0-11.0 \mathrm{~Hz}$; alfa2, $11.0-14.0$ $\mathrm{Hz}$; beta1, $14.0-25.0 \mathrm{~Hz}$ y beta2, $25.0-25.0 \mathrm{~Hz}$. Todas las bandas de la actividad EEG fueron correlacionadas con los puntajes obtenidos del WCST.

\section{Análisis estadístico}

El análisis estadístico fue realizado con el programa STATISTICA para Windows 7 (StatSoft, Inc). Antes de la aplicación del procedimiento estadístico, se comprobó la distribución normal de los datos por medio de la prueba de Levene. Se utilizó la prueba de Sperman para correlacionar los puntajes de la prueba WCST con las bandas de la actividad EEG. El nivel de significancia aceptado fue de $p<0.05$.

\section{Resultados}

\section{Características de las participantes}

La edad promedio de las mujeres participantes fue de 51.5 años, con un índice de masa corporal de 30.10, 6 años de escolaridad, 4 años de inicio de la menopausia, presión arterial y niveles de glucosa dentro de los rangos normales.

\section{Análisis de correlación}

En la tabla 1 se muestran los coeficientes de correlación entre los puntajes de la prueba WCST y las variables de la actividad EEG. El número de categorías completadas se correlacionó positivamente con la banda de beta $2(r=0.36, p=0.04)$, el número de errores perseverativos se correlacionó negativamente con la banda de beta $2(r=-0.37, p=0.03)$ y el número de errores cometidos se correlacionó negativamente con la banda de beta2 $(r=-0.37, p=0.03)$. El resto de las variables no se correlacionaron con la actividad EEG.

\section{Discusión}

El presente estudio provee de evidencia de que la actividad EEG registrada en reposo se correlaciona con las funciones ejecutivas medidas por la prueba WCST en mujeres en la posmenopausia. La actividad EEG en reposo es un índice dinámico de activación cortical y función cognitiva y por eso puede ser considerado un fenotipo intermediario para muchas conductas en la que está implicada la activación. Las señales $E E G$, que reflejan diferencias en los potenciales eléctricos en las dendritas neuronales de las células piramidales de la corteza cerebral, varían continuamente, aun cuando el cerebro esté en reposo (Steriade, 1993). Las mujeres sanas en la posmenopausia analizadas en el presente estudio mostraron correlaciones entre la actividad EEG y los puntajes de la prueba WCST. El número de categorías completadas, un indicador de la buena ejecución de la prueba, se correlacionó positivamente con la actividad de la banda de beta2, mientras que el número de errores perseverativos y errores de la prueba se correlacionaron inversamente, sugiriendo una influencia en la modulación de las funciones ejecutivas. La prueba WCST demanda asignar recursos, enfocar atención hacia procesos cognitivos internos, decisión, planeación del siguiente movimiento apropiado, basado en la experiencia previa, y memoria de trabajo, todas ellas funciones ejecutivas atribuidas a la corteza prefrontal (Stuss, Benson, Clermont, Della Malva, Kaplan \& Weir, 1986; Goldman-Rakic, 1987; Fuster, Bauer \& Jervey, 1985; Petrides, 1991; Damasio, 1993). La actividad beta se ha encontrado en diferentes condiciones de alerta incrementada (Steriade, 1993), 
en tareas cognitivas que requieren de movimientos finos de los dedos y atención focalizada (Murthy \& Fetz, 1992), reactividad enfocada previa a la ejecución de una tarea cognitiva compleja (Sheer, 1984), ejecución ejecutiva (Solis-Ortiz, Guevara, M.A. \& Corsi-Cabrera, 2004) y tareas cognitivas de memoria visual (Krause, Pesonen \& Hämäläinen, 2010).

En el presente estudio, un grupo de mujeres fue analizada en la posmenopausia, caracterizada por un decremento de estrógenos (Santoro \& Tortoriello, 1999) que puede afectar la función cognitiva (Sherwin, 2003). Sin embargo, el estudio encontró que las categorías completadas en el WCST, indicador de buena función ejecutiva, se correlacionó positivamente con actividad EEG rápida (beta2), indicando un estado de activación adecuado y necesario para focalizar la atención y disminuir los errores, y lograr una ejecución exitosa. En conclusión, los resultados del presente estudio indican que un estado de activación cortical de frecuencias rápidas es necesario para ejecutar pruebas que involucren funciones ejecutivas en mujeres con bajos niveles de estrógenos debidos a la posmenopausia.

\section{Agradecimientos}

Este trabajo fue financiado parcialmente por el CONACYT 060645 y la Universidad de Guanajuato. Mayra Gutiérrez-Muñoz recibió la beca de Maestría por el CONACYT 204424 y una becatesis por parte del CONCYTEG 08-16-k119-058.

\section{Conflicto de intereses}

Los autores declaran que no existen conflictos de intereses.

\section{Referencias}

Becker, D., Creutzfeldt, O.D., Schwibbe, M. \& Wuttke, W. (1982). Changes in physiological, EEG and psychological parameters in women during the spontaneous menstrual cycle and following oral contraceptives. Psychoneuroendocrinology, 7, 75-90.

Berman, K.F., Ostrem, J.L., Randolph, C., Gold, J., Goldberg, T.E., Coppola, R., et al. (1995). Physiological activation of a cortical network during performance of the Wisconsin Card Sorting Test: a positron emission tomography study. Neuropsychologia, 33, 1027-1046.

Buzsáki, B. (2006). Rhythms of the Brain. Madison, New York: Oxford University Press.

Corsi-Cabrera, M., Solís-Ortiz, S. \& Guevara, M.A. (1997). Stability of EEG inter-and intrahemispheric correlation in women. Electroencephalography and Clinical Neurophysiology, 102, 248-255.

Corsi-Cabrera, M., Galindo-Vilchis, L., del-Río-Portilla, Y., Arce, C. \& RamosLoyo, J. (2007). Within-subject reliability and inter-session stability of EEG power and coherent activity in women evaluated monthly over nine months. Clinical Neurophysiology, 118, 9-21.

Damasio, A.R., Tranel, D. (1993). Nouns and verbs are retrieved with differently distributed neural systems. Proceeding of the National Academy of Science of the United State of America, 90, 4957-4960.

Folstein, M. F., Folstein, S. F. \& McHugh, P. R. (1975). Mini-mental state: A practical method for grading the cognitive state of patients for the clinician. Journal Psychiatry Research, 12, 189-198.

Fuster, J. M., Bauer, R. H. \& Jervey, J.P. (1985). Functional interactions between inferotemporal and prefrontal cortex in a cognitive task. Brain Research, 330, 299-307.

Goldman-Rakic, P.S. (1987). Development of cortical circuitry and cognitive function. Child Development, 58, 601-622.

Halbreich, U., Lumley, L. A., Palter, S., Manning, C., Gengo, F. \& Joe, S.H. (1995). Possible acceleration of age effects on cognition following menopause. Journal Psychiatry Research, 29, 153-163.

Heaton, R. K. (1981). Manual for the Wisconsin Card Sorting Test. Odessa, Florida: Psychological Assessment Resources.
Heaton, R. K., Chelune, G. J., Taley, J. L., Kay, G.G. \& Curtiss, G. (1993). Wisconsin Card Sorting Test Manual (Revised and expanded). Odessa, Florida: Psychological Assessment Resources.

Krause, C.M., Pesonen, M. \& Hämäläinen, H. (2010). Brain oscillatory 4-30 Hz

electroencephalogram responses in adolescents during a visual memory task. Neuroreport, 21, 767-771.

Murthy, V.. N. \& Fetz, E.E. (1992). Coherent 25-35 Hz oscillations in the sensorimotor cortex of the awake behaving monkey. Proceedings of National Academy of Sciences of the United States of America, 89, 5670-5674.

Nauta, W. J. (1971). The problem of the frontal lobe: a reinterpretation. Journal of Psychiatry Research, 8, 167-87.

Niedermeyer, E. (1993). EEG patterns and genetics. En Niedermeyer, E. \& Lopes Da Silva F. (eds.) Electroencephalography. Basic Principles, Clinical Applications, and Related Fields (pp. 192-195). Baltimore, Maryland, USA: Williams \& Wilkins.

Petrides, M. (1991). Functional specialization within the dorsolateral frontal cortex for serial order memory. Proceedings Biological Sciences /The Royal Society, 246, 299-306.

Reilly, E. L. (1993). EEG recording and operation of the apparatus. En Niedermeyer, E. \& Lopes Da Silva F. (eds.) Electroencephalography. Basic Principles, Clinical Applications, and Related Fields (pp. 104-124). Baltimore, Maryland, USA: Williams \& Wilkins

Salinsky, M., Oken, B. \& Morehead, L. (1991). Test-retest reliability in EEG frequency analysis. Electroencephalography and Clinical Neurophysiology, 79, 382392.

Santoro, N. \& Tortoriello, D. V. (1999). Endocrinology of the Climacteric. En Seifer, D.B. \& Kennard, E. A. (Eds.) Menopause. Endocrinology and Management. (pp. 21-34). Totowa, New Jersey: Humana Press.

Sepúlveda Angulo, M. T., Pérez Luque, E., \& Solís-Ortiz, S. (2009). Atención sostenida asociada con hormonas ováricas en mujeres en la premenopausia y posmenopausia. Revista Chilena de Neuropsicología, 4, 149-159.

Sheer, D. (1984). Focused arousal, $40 \mathrm{~Hz}$, and dysfunction. En Ebert T. (ed.) Selfregulation of the Brain and Behavior (pp. 64-84). Berlin, Germany: Springer.

Sherwin, B.B. (2003). Estrogen and cognitive functioning in women. Endocrine Reviewer, 24, 133-151.

Solís-Ortiz, S., Ramos, J., Arce, C., Guevara, M.A. \& Corsi-Cabrera, M. (1994). EEG oscillations during menstrual cycle. International Journal Neuroscience, 79, 279-292.

Solís-Ortiz, M.S., Guevara, M.A. \& Corsi-Cabrera, C.M. (2004). Performance in test demanding prefrontal functions is favored by early luteal phase progesterone: an electroencephalographic study. Psychoneuroendocrinology, 29, 1047-1057.

Steriade, M. (1993). Cellular substrates of brain rhythms. En Niedermeyer, E. \& Lopes Da Silva, F. (eds.) Electroencephalography. Basic Principles, Clinical Applications, and Related Fields (pp. 27-62). Baltimore, Maryland: Williams \& Wilkins.

Stuss, D. T., Benson, D. F., Clermont, R., Della Malva, C. L., Kaplan, E. F. \& Weir, W. S. (1986). Language functioning after bilateral prefrontal leukotomy. Brain Language, 28, 66-70.

Sullivan, M. E. \& Fugate, W. N. (2001). Midlife women's attributions about perceived memory changes: observations from the Seattle Midlife Women's Health Study. Journal Women's Health Gender Based Medical, 10, 351-362.

Tivis, L. (1999). Alterations in cognitive function in menopause. En Seifer, D.B. \& Kennard, E.A. (eds.) Menopause: Endocrinology and Management (pp. 97-110). Totowa NJ: Human Press. 